\title{
ИСХОДЫ ЛЕЧЕНИЯ ПАЦИЕНТОВ С ПРОЛАКТИН-СЕКРЕТИРУЮЩИМИ АДЕНОМАМИ ГИПОФИЗА: ДЛИТЕЛЬНОЕ НАБЛЮДЕНИЕ ЗА 40 ПАЦИЕНТАМИ
}

\author{
Сакеян И.С., Цой У.А., Гринева Е.Н.
}

ФГБУ «НМИЦ им. В. А. Алмазова» Минздрава России, Санкт-Петербург

ЦЕЛЬ: изучить клинические особенности и результаты стационарного лечения пациентов с пролактин-секретирующими аденомами гипофиза.

МАТЕРИАЛЫ И МЕТОДЫ: проведен ретроспективный анализ 40 пациентов с пролактиномами, госпитализированных в НМИЦ им. В.А. Алмазова в период с июня 2014г. по январь 2020 г., оценены характер и длительность терапии, уровень пролактина и данные МРТ в динамике.

РЕзУЛЬТАТЫ: сорок пациентов (18 (45\%) мужчин, 22 (55\%) женщины)) были включены в исследование. У восьми пациентов (20\%) отмечались микропролактиномы и 32 (80\%) - макропролактиномы. Имели место следующие причины госпитализации: 32 (80\%) пациента были направлены из разных регионов Российской Федерации из-за неэффективности проводимой терапии, 8 (20\%) были переведены из отделения нейрохирургии после транссфеноидальной аденомэктомии (ТСА). 11 (27,5\%) пациентов находились на терапии каберголином в дозе менее 2 мг/нед, что привело к нормализации уровня пролактина и уменьшению объема аденомы. 16/40 (40\%) пациентов получали каберголин в дозе более 2 мг/нед без нормализации уровня пролактина и уменьшения объема аденомы. Среди этих 16 пациентов у 8 доза каберголина была увеличена (до 3,5-4,5 мг/нед), у 4 из них уровень пролактина в результате нормализовался. У двух пациентов каберголин был заменен на бромокриптин (7,5-10 мг/сут), у одного пациента это привело к нормализации уровня пролактина. ТСА выполнена у 6/16 (38\%) пациентов; однако операция не была успешной в 3 случаях (нормализация уровня пролактина после отсутствовала). Десяти из 40 пациентов (25\%) были выполнены ТСА до достижения максимальной переносимой дозы каберголина/бромокриптина; только у трех из них имели место срочные показания к оперативному вмешательству. У одного пациента лечение каберголином было прекращено из-за аллергической реакции, после чего выполнена ТСА, приведшая к нормализации уровня пролактина. У 2 из 40 пациентов на фоне терапии бромокриптином (7,5-12,5 мг/сут) произошли нормализация пролактина и уменьшение объема аденомы.

ВЫВОдЫ: с целью снижения количества госпитализаций пациентов с пролактиномами и повышения эффективности консервативной терапии необходимо выполнять увеличение доз агонистов дофаминовых рецепторов до максимально переносимых либо смену неселективных и селективных препаратов на амбулаторном этапе. При отсутствии достижения эффекта целесообразна госпитализация, в том числе для решения вопроса о целесообразности оперативного лечения.

КЛЮЧЕВЫЕ СЛОВА: пролактиномы; бромокриптин; каберголин; резистентность. 